\title{
Time-Dependent Changes in Mechanical Properties of Carbon Fiber Vinyl Ester Composites Exposed to Marine Environments
}

\author{
Arash Afshar $^{\mathrm{a}, *}$, Heng-Tseng Liao ${ }^{\mathrm{b}}$, Fu-pen Chiang ${ }^{\mathrm{b}}$, Chad S. Korach ${ }^{\mathrm{c}}$ \\ ${ }^{a}$ Department of Mechanical Engineering, Saginaw Valley State University, University Center, MI, USA \\ ${ }^{\mathrm{b}}$ Department of Mechanical Engineering, Stony Brook University, Stony Brook, NY, USA \\ ${ }^{\mathrm{c}}$ Department of Engineering, University of Mount Union, Alliance, OH, USA
}

\begin{abstract}
:
Carbon fiber-vinyl ester (CFVE) composites are good candidates for use in civil and naval vessels. CFVE composites offer high strength and stiffness-to-weight ratio and superior resistance to electrochemical corrosion. However, during application CFVE composites are exposed to harsh marine environments which can significantly degrade the mechanical properties. Here, the time dependent changes in flexural mechanical properties of CFVE composites due to exposure from marine environments are investigated. Exposure to marine environments are simulated by indoor water immersion, accelerated aging in environmental chambers, and outdoor exposure in a tidal pond. Comparison between the conditions will be discussed in the context of long-term outdoor exposure with controlled laboratory conditions.
\end{abstract}

\section{Introduction:}

A wide range of marine environmental elements can adversely affect the mechanical properties of fiber-reinforced polymeric composites including humidity, sea water, thermal variations, and ultraviolet radiation, to name a few. To employ fiber reinforced composites in naval and civil vessels, a detailed characterization of the effects of marine environments on mechanical properties of the materials is essential.

\footnotetext{
* Corresponding author.

E-mail address: aafshar@svsu.edu (A. Afshar).
} 
The effect of moisture on the mechanical properties of fiber-reinforced polymeric composites has been investigated in recent years $[1,2,3]$. Moisture can be absorbed into the fiber-matrix interface and replace or weaken resin/fiber chemical bonds. Moisture reduces the strength of the fiber-matrix interface via irreversible hydrolysis and plasticization. In composite materials with a brittle matrix, the damage mode can change from being matrix-dominated before moisture absorption to a fiber-matrix interfacial mode after saturation. This leads to a significant change in the strength of composites. In composite materials with a very brittle matrix, the moisture's plasticizing effect may cause a small increase in the material toughness $[4,5]$. Different water chemistry effects the moisture absorption of composite materials. The weight gain of specimens immersed in sea water is lower than that of ones immersed in pure water. This is due to the fact that polymers can be considered as a semi-permeable membrane and the osmotic pressure reduces the driving force for moisture absorption. The hydrostatic pressure in deeply immersed specimens may not have a significant effect on the quantity of moisture absorption. The increased driving force for moisture absorption due to high pressure will be eliminated by the simultaneous reduction in free volume by hydrostatic pressure $[4,5]$.

Ultraviolet (UV) radiation is another environmental factor in degrading polymeric composite materials $[6,7,8]$. UV light is not able to penetrate deep into fiber-reinforced composite laminates, and as a result, most of the matrix remains undamaged and fibers maintain their bonds to the matrix. Composite stiffness varies slightly due to UV radiation, as the change induced by UV radiation is a surface phenomenon, while modulus is a bulk material property. In many polymers, UV irradiation initiates chain scissions on the exposed surfaces, which produce small free molecules. These molecules are capped by oxygen and can be removed from the specimen, resulting in polymer weight loss [9]. The energy of UV photons is almost the same as the energy 
of polymer covalent bonds, which is about $290-460 \mathrm{~kJ} / \mathrm{mole}$. UV photons initiate photo-oxidative reactions, which deteriorate polymers by changing their chemical structures. Molecular chain scission and/or chain cross-linking are typically produced by photo-oxidative reactions. Chain scission reduces the molecular weight of the polymer, and lowers strength and heat resistance. Chain cross-linking increases the brittleness of the polymer, which may result in microcracking $[10,11]$.

Combined exposure to UV radiation and moisture from environmental conditions synergistically exacerbates composite property degradation and has gained much attention in recent years. The microcracks at the UV-irradiated surface of polymeric composites, can be propagated into the laminates in the presence of thermal variations [9]. The microcracks can also facilitate the ingression of moisture into the laminate. Moisture is able to dissolve and remove products of photo-oxidation reactions from a UV-irradiated surface and provide a fresh surface for further degradation by UV radiation $[11,12,13]$. The effects of combined exposure on the mechanical properties can be more deleterious compared to individual exposures. The transverse strength is the most susceptible property of polymeric composites to environmental elements and may decrease substantially after long-time exposures [11].

CFVE composites are promising candidates for naval and marine applications $[14,15,16]$. Carbon fibers are hydrophobic materials and aqueous environments have a minimal effect on the mechanical properties. Vinyl ester exhibits high corrosion resistance as well as superior resistance against water absorption. To employ CFVE composites in marine environments, it is necessary to characterize time-dependent changes in the mechanical properties and also to seek a method to approximate real-life outdoor exposures with indoor controlled conditioning. This 
provides valuable property values to predict the life expectancy of CFVE composites in harsh marine environments.

\section{Materials:}

Carbon-fiber reinforced vinyl ester unidirectional composite laminates (Graphtek LLC) were used for all sets of experiments and conditions. Laminates have an isotropic vinyl ester matrix with a modulus of $3.2 \mathrm{GPa}$ and carbon fibers with longitudinal and transverse moduli of $220 \mathrm{GPa}$ and $22 \mathrm{GPa}$, respectively. Laminates have a fiber volume fraction of 0.56 , and consist of 8 plies. Composite laminate sheets with nominal thickness of $1.4 \mathrm{~mm}$ were machined using a diamond wet saw into $12.5 \times 77 \mathrm{~mm}$ (width $\times$ length) coupons. Two sets of unidirectional specimens were prepared, to represent the two material principal directions of the laminate. The two sets are referred to, based on fiber orientation, as $\left[0^{\circ}\right]$ and $\left[90^{\circ}\right]$. For the former (i.e., longitudinal) the fibers are aligned with the length of specimens (i.e., the $77 \mathrm{~mm}$ long side), while for the latter (i.e., transverse) the fibers are aligned with the width of specimens (i.e., the $12.5 \mathrm{~mm}$ long side).

\section{Exposure Conditions:}

Sample coupons were divided into three groups. The first group of samples $\left(\left[0^{\circ}\right]\right.$ and $\left.\left[90^{\circ}\right]\right)$ were exposed up to 2000 hours of combined and individual accelerated aging using three chambers: i) Moisture and heat in a Tenney Benchmaster BTRS temperature and humidity chamber, ii) UV Radiation in a Q-Lab QUV/se accelerated weathering chamber, and iii) Salt spray and heat in a Bemco P700XL chamber. UV radiation simulates natural sunlight using fluorescent UV bulbs at a $340 \mathrm{~nm}$ wavelength. Intensity is monitored by real-time UV irradiance sensors. One-half the samples in the first group were exposed to individual exposures and the other half were rotated every 48 hours between two sets of chambers: salt spray and UV chambers, and heat and humidity and UV chambers to produce combined exposures. Because UV radiation exposure is 
only on one side of the coupon in the QUV/se chamber, sample sides were rotated every 24 hours to receive uniform UV radiation. In the temperature and humidity chamber, moisture was set at $85 \%$ relative humidity $(\mathrm{RH})$ and temperature at $35 \mathrm{C}$. In the QUV chamber, the UV irradiance was set at $0.6 \mathrm{~W} / \mathrm{m}^{2}$ at $60 \mathrm{C}$. The salt spray chamber was fed with $5 \% \mathrm{NaCl}$ solution at constant flow rate using $\sim 70 \mathrm{kPa}$ air pressure and with a chamber temperature set to $35 \mathrm{C}$. The conditions in the chambers remained constant for the duration of exposures.

The second group of samples (all $\left[0^{\circ}\right]$ ) were immersed in distilled water, salt water, and sea water for a one year duration at room temperature, in closed containers. The third group of samples (all $\left[0^{\circ}\right]$ ) were mounted on a ladder frame with four different levels which was placed in a tidal pond for one year. Based on the position of samples on the ladder frame, they were exposed to varying amounts of sea air (which contains sunlight and atmospheric moisture) and sea water. The four levels of the frame are as follows: The upper level (Row 1) samples always remained out of water and only exposed to the sea air for the entire duration. The samples in the second level (Row 2) were exposed to 18 hours of sea air and 6 hours of sea water immersion during each day; two cycles of 9 hours sea air followed by 3 hours sea water. The samples in the third level (Row 3) were exposed to 6 hours of sea air and 18 hours of sea water immersion for each day; two cycles of 3 hours sea air followed by 9 hours sea water. The samples in the lower level of the frame (Row 4) were immersed for the entire duration in sea water. Five specimens per condition were used in the experiments.

\section{Experimental setup:}

As a result of the samples exposure to moisture and photo-degradation conditions, the samples mass will change during the exposure. The mass of the composite samples were measured using a precision balance at every 48 hours of exposure for the environmental chambers. In the case of 
combined exposure to moisture and UV radiation, mass of the samples were tracked at the end of the UV cycles, such that condensate was not present on the samples surfaces. Three point bending tests were performed on the composite coupons following the ASTM D790 standard [17] using a screw-driven mechanical loading frame (TiraTest 26005) with a $0.5 \mathrm{kN}$ load cell. Support geometry followed ASTM D790, with the support span set for $60 \mathrm{~mm}$, resulting in a span/thickness ratio of $\sim 43$. A crosshead rate of $4.25 \mathrm{~mm} / \mathrm{min}$. was used to give a strain rate of $0.01 \mathrm{~mm} / \mathrm{min}$. The morphology of the fracture surfaces of broken samples from three-point bending test were examined next using a scanning electron microscope (Hitachi, HD2700C).

\section{Results and Discussion:}

\section{1-Time-Dependent Weight Change:}

Weight measurements of composite specimens were performed every 48 hours during exposure to salt spray and combined UV and salt spray from the environmental chambers. Percent weight change was calculated by taking the difference between the original average sample weight and the average measured weight at each measurement time, divided by the average original weight and multiplied by 100 . The results indicate that samples constantly exposed to salt spray had mass gain, reaching a steady-state due to moisture uptake $(\sim 1 \%)$. There was a significant difference between the specimens in the combined UV-salt spray condition versus those in the constant moisture condition, particularly for the salt spray cases (Figure 1). The decrease in the weight after a peak value of $\sim 700$ hours for the combined UV-salt spray cases indicates loss of surface material due to degradation and erosion of the matrix. It is worth noting that no change in volume was detected during exposure of samples to salt spray condition. For the combined exposure to UV and salt spray, no significant volume change occurred during the first 700 hours 
of exposure. For the rest of the exposure time, the decrease in the volume was proportional to the weight loss in samples.

\section{2- Time-Dependent Flexural Response:}

Samples exposed to different environmental exposures were characterized by three-point bending test to determine the flexural modulus and strength. All exposure conditions show an insignificant effect on longitudinal flexural modulus of specimens when compared to flexural modulus of virgin (unexposed) specimens, and within experimental error (Figures 2). The maximum decrease in the longitudinal flexural modulus occurred for specimens exposed to 2000 hours combined UV radiation and salt spray in environmental chambers $(-7 \%)$. Results demonstrate that the longitudinal flexure strength decreases significantly for different types of exposure (Figure 3). There is minimal difference between residual flexural strength of specimens immersed in distilled, salt and sea water. Furthermore, the specimens' longitudinal flexural properties were almost identical for both 6 month and one year exposure durations. This implies that specimens were almost saturated with moisture after 6 months exposure and extending the exposure duration will not change the moisture content of specimens. The significant decrease in longitudinal flexural strength due to moisture absorption is attributed to degradation of the fibermatrix interface, causing swelling and loss of integrity in carbon fiber vinyl ester composites. Outdoor exposures to combined UV and moisture (i.e. sea air or sea water) produce higher degradation in flexural strength for longer exposure durations. UV radiation produces microcracks on the surface of irradiated specimens by altering their chemical structures, accelerating the diffusion of moisture into the composite laminates. Moisture dissolves and removes the damaged materials on irradiated surfaces and provides fresh surfaces for further degradation by UV radiation. 6 month outdoor immersion in sea water reduced the longitudinal 
flexural properties as the same amount as six months or one year indoor immersions. Unlike indoor immersion, extending the duration of outdoor immersion in sea water continuously decreases the longitudinal flexural strength of specimens. This can be attributed to effects of marine organisms, temperature fluctuations and other uncontrolled environmental conditions. For all types of conditioning in environmental chambers, a continuous and gradual reduction in longitudinal flexural strength were observed during exposures. This result is believed to be due to the elevated temperatures in the laboratory chambers versus the indoor immersion temperature (21C). 2000 hours exposure to combined UV, heat and humidity in environmental chambers produced the same amount of damage in longitudinal flexural strength as 1 year outdoor exposure to UV and sea air (Row1). Similarly, 2000 hours exposure to combined UV and salt water in environmental chambers decreased the longitudinal flexural strength as the same amount as 1 year outdoor exposure to UV, sea air and mostly sea water (Row 3). Results imply that 2000 hours combined exposures to UV and moisture (i.e. humidity or salt water) in environmental chambers is comparable to 1 year outdoor exposure to combined UV and moisture (i.e. sea air or sea water) in a tidal pond. The effect of marine environments on transverse flexural properties of CFVE is shown in Figure 4. Significant decreases are observed in transverse flexural modulus and flexural strength of specimens exposed to different marine conditions in environmental chambers. The maximum decreases in transverse flexural modulus $(-14.6 \%)$ and transverse flexural strength $(-33.5 \%)$ were observed in specimens exposed to UV and salt spray for 2000 hours. For all types of exposures in environmental chambers, higher damage in transverse flexural strength were observed in the first 800 hours of exposure time as compared to the rest of the exposure duration. This can be attributed to loss in fiber-matrix interface toughness due to significant moisture uptake and extensive UV-induced microcracks on 
the surface of specimens during first stage of exposure (800 hours). On the other hand, for combined exposure to UV and moisture (i.e., humidity or salt spray), an insignificant loss in transverse flexural modulus was observed during the first 800 hours exposure time. This is due to the fact that laminates' thickness starts to decrease continuously due to the erosion of UVirradiated surfaces after about 700 hours exposure to combined UV and moisture (Figure 1), resulting in significant decrease in transverse flexural modulus during longer exposure times. This is not the case for transverse flexural modulus of specimens exposed to individual exposures (heat and humidity or salt spray), since no significant surface erosion occurs for these types of exposures.

\section{3-Fracture Surface Microscopy:}

The fracture surface of $\left[90^{\circ}\right]$ specimens were characterized after 2000 hours exposure to different marine environmental conditions by utilizing Scanning Electron Microscopy (Figure 5). All images were captured near to the exposed surfaces of the specimens. The images provide evidence of moisture ingression and its subsequent damage to the vinyl ester resin, which is shown by discolored regions near the surface of laminates. Figure 5a shows the fracture surface of composite laminates in pristine condition (i.e. no environmental exposure). Figures $5 \mathrm{~b}$ and $5 \mathrm{c}$ exhibit damaged layers on the surface of composite laminates after exposure to salt spray and heat and humidity, respectively. In the case of heat and humidity exposure, moisture penetrated through the materials to a greater depth due to the elevated temperature and produced small cavities in the vinyl ester resin. The most severe damage was observed in the case of combined exposure to UV and heat and humidity shown in Figure 5d. This is due to the fact that UV produces microcracks on the surface of the laminates, providing passages for moisture penetration into composite laminates. Figure 5e shows a magnified image of the damaged area in 
Figure 5d. It can be seen that fibers completely lost their confinement with the matrix and therefore they are not able to contribute in load transfer between fibers and matrix, resulting in a substantial decrease in flexural strength of specimens. Figures $5 \mathrm{~b}$ to $5 \mathrm{e}$ demonstrate that extensive damage occurs in a thin layer on the laminate surface due to environmental exposures. This significantly decreases the flexural properties of the laminate, which is primarily dependent on the load bearing capacity of the outer layers of the laminate. Due to this fact, the same magnitude of decrease in flexural properties will be expected for environmentally exposed specimens of different thicknesses or widths.

\section{Conclusion:}

Carbon fiber-reinforced vinyl ester unidirectional composite laminates were characterized after exposure to different marine environments: Long-term outdoor exposure to UV, sea air and sea water in a tidal pond; indoor immersion in distilled, salt and sea water; and indoor exposure to UV radiation, salt spray, and heat and humidity in accelerated weathering chambers. The study shows that the effect of different types of exposure on longitudinal flexural modulus is not significant, though the transverse modulus has significant decreases. However, flexural strength has been diminished significantly due to environmental exposures for both the longitudinal and transverse directions. This can be explained by the loss in fiber-matrix interface toughness due to moisture absorption as well as loss in integrity on the surface of laminates due to extensive UV-induced microcracks, which can synergistically or individually diminish out-of-plane load bearing capacity and deteriorate the load transfer mechanism between matrix and fibers. Comparison between long-term outdoor exposure, indoor immersion and accelerated laboratory conditions, demonstrated that 2000 hours combined exposure to UV and moisture in environmental chambers is comparable to one year outdoor exposure in a tidal pond. Transverse 
flexural properties of carbon fiber vinyl ester composites are more sensitive to marine environments compared to longitudinal flexural properties, since transverse flexural properties are primarily matrix-dominated which undergo significant degradation during environmental exposure. Results of the time-dependent property changes of the CFVE composites exposed to marine environments is important for design of vessels and structures used for long durations that may undergo aging due to exposure conditions, and provides guidelines for the comparison of laboratory testing conditions to outdoor exposure.

\section{Acknowledgments:}

The authors respectfully acknowledge the support from Drs. Yapa D.S. Rajapakse and Airan J. Perez from the Office of Naval Research through grant N00014-11-1081.

\section{References:}

[1] Y.Weitsman, M. Elahi, "Effects of fluids on the deformation, strength and durability of polymeric composites-an overview," Mech Time-Dependent Mater, Vol. 4, pp. 107-126, 2000.

[2] Y. Weitsman, A. In Kelly, C. Zeben (eds.), "Effects of fluids on polymeric compositesareview," Comprehensive Composite Materials, R. Talreja, J.-A.E. Manson (eds.), Polymeric Matrix Composites, Elsevier, Amsterdam, Vol. 2, pp. 369-401, 2000.

[3] J. P. Komorowski, "Hygrothermal effects in continuous fibre reinforced composites. Part I: Thermal and moisture diffusion in composite materials," National Aeronautical Establishment, National Research Council, Canada. Aeronautical Note NAE-AN-4, NRC No. 20974; January 1983.

[4] T. S. Grant and W. L. Bradley, "In-Situ Observations in SEM of Degradation of Graphite/Epoxy Composite Materials due to Seawater Immersion," Journal of Composite Materials, Vol. 29, pp. 852-867, 1995.

[5] F. McBagonluri, K. Garcia, M. Hayes, K. N. E. Verghese, J. J. Lesko , "Characterization of fatigue and combined environment on durability performance of glass/vinyl ester composite for infrastructure applications," International Journal of Fatigue, Vol. 22, pp. 53-64, 2000. 
[6] Ricky S.C. Woo, Yanghai Chen, Honggang Zhu, Jing Li, Jang-Kyo Kim, Christopher K.Y. Leung, "Environmental degradation of epoxyorganoclay nanocomposites due to UV exposure. Part I: Photo-degradation," Composites Science and Technology, Vol. 67, pp. 34483456, 2007.

[7] Ricky S.C. Woo, Honggang Zhu, Christopher K.Y. Leung, Jang-Kyo Kim, "Environmental degradation of epoxy-organoclay nanocomposites due to UV exposure: Part II residual mechanical properties," Composites Science and Technology, Vol. 68, pp. 2149-2155, 2008.

[8] Andrew W. Signor, Mark R. VanLandingham, Joannie W. Chin, "Effects of ultraviolet radiation exposure on vinyl ester resins: characterization of chemical, physical and mechanical damage," Polymer Degradation and Stability, Vol. 79, pp. 359-368, 2003.

[9] W.B. Liau and F. P. Tseng, "The Effect of Long-Term Ultraviolet Light Irradiation on Polymer Matrix Composites," Vol. 19, pp. 440-445, 1998.

[10] B. Ranby and J.F. Rabek, "Photodegradation, Photo-Oxidation and Photostabilization of Polymers," John Wiley and Sons, London, 1975.

[11] Bhavesh G. Kumar, Raman P. Singh and Toshio Nakamura, "Degradation of Carbon Fiberreinforced Epoxy Composites by Ultraviolet Radiation and Condensation," Journal of Composite Materials, Vol. 36, No. 24, pp. 2713-2733, 2002.

[12] C.S. Korach, A. Afshar, H.-T. Liao, and F.-P. Chiang, "Comparison of sea water exposure environments on the properties of carbon fiber vinylester composites," In Challenges in Mechanics of Time-Dependent Materials and Processes in Conventional and Multifunctional Materials, ed: Springer. 2014;2:139-44.

[13] Raman P. Singh, Mikhail Khaitb, Suraj Zunjarrao, Chad S. Korach, and Gajendra Pandey, "Environmental Degradation and Durability of Epoxy/Clay Nanocomposites," Journal of Nanomaterials, Vol. 2010, ID: 352746, 2010.

[14] Arash Afshar, Maen Alkhader, Chad S. Korach, Fu-pen Chiang, "Effect of long-term exposure to marine environments on the flexural properties of Carbon Carbon Fiber Vinylester Composites," Journal of composite structures, Vol. 126, 72-77, 2015.

[15] Arash Afshar, Maen Alkhader, Chad Korach and Fu-pen Chiang, "Synergistic Effects of Fatigue and Marine Environments on Carbon fiber Vinyl-Ester Composites," Journal of Engineering Materials and Technology, Vol. 137 (4), 041002-041002, 2015.

[16] Arash Afshar, "Effects of marine environments and cyclic loading on carbon fiber vinylester composites," Stony Brook University; 2014.

[17] D790, A., 2000, "Standard Test Method for Flexural Properties of Unreinforced and Reinforced Plastics and Electrical Insulating Materials." 


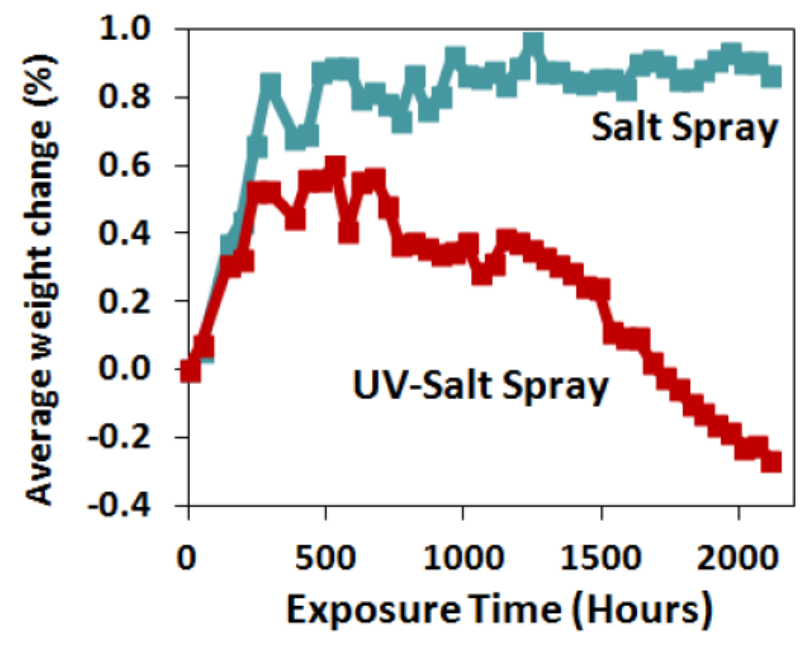

Figure 1: Average sample weight change for CFVE specimens

exposed to constant salt spray and combined UV-salt spray 

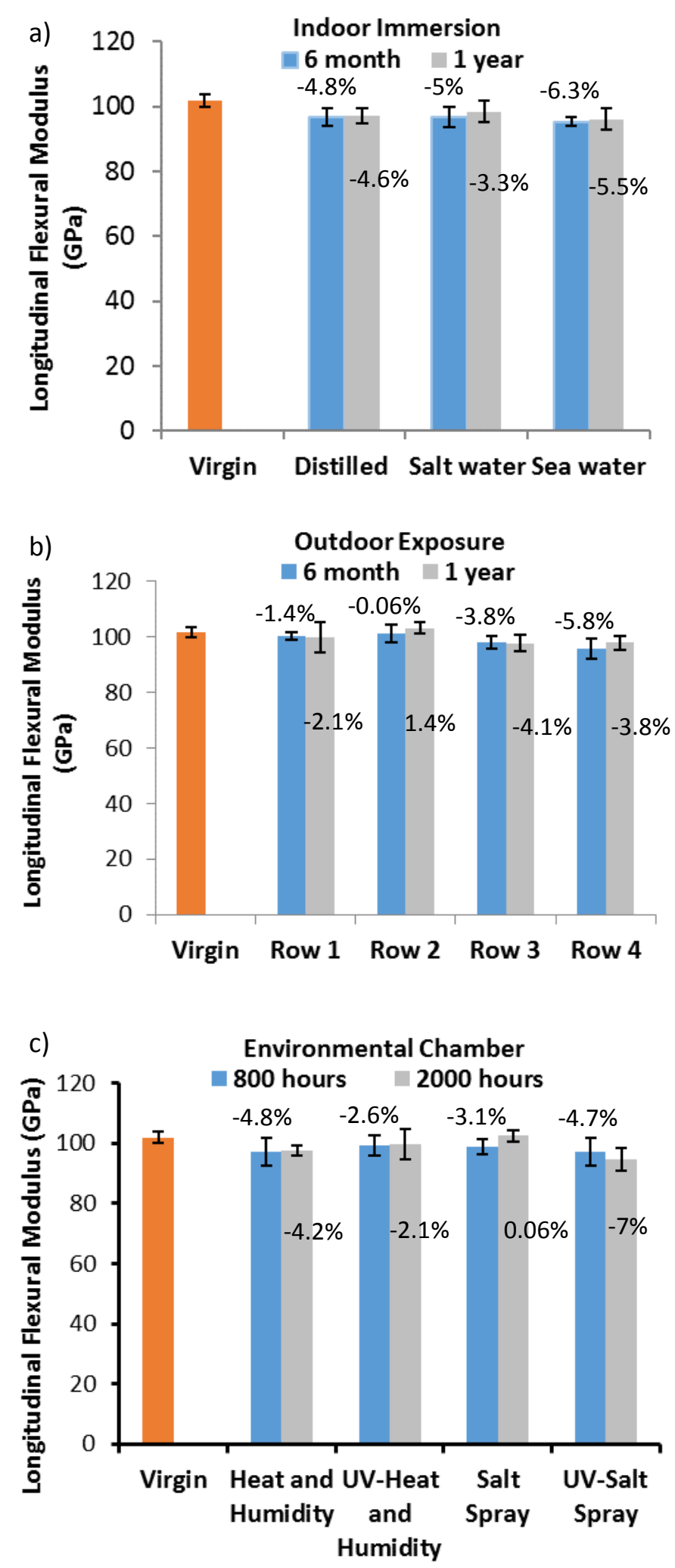

Figure 2: Longitudinal flexural modulus of CFVE composites exposed to (a) Indoor immersion, (b) Outdoor environmental exposure, and (c) Conditioning in environmental chambers 

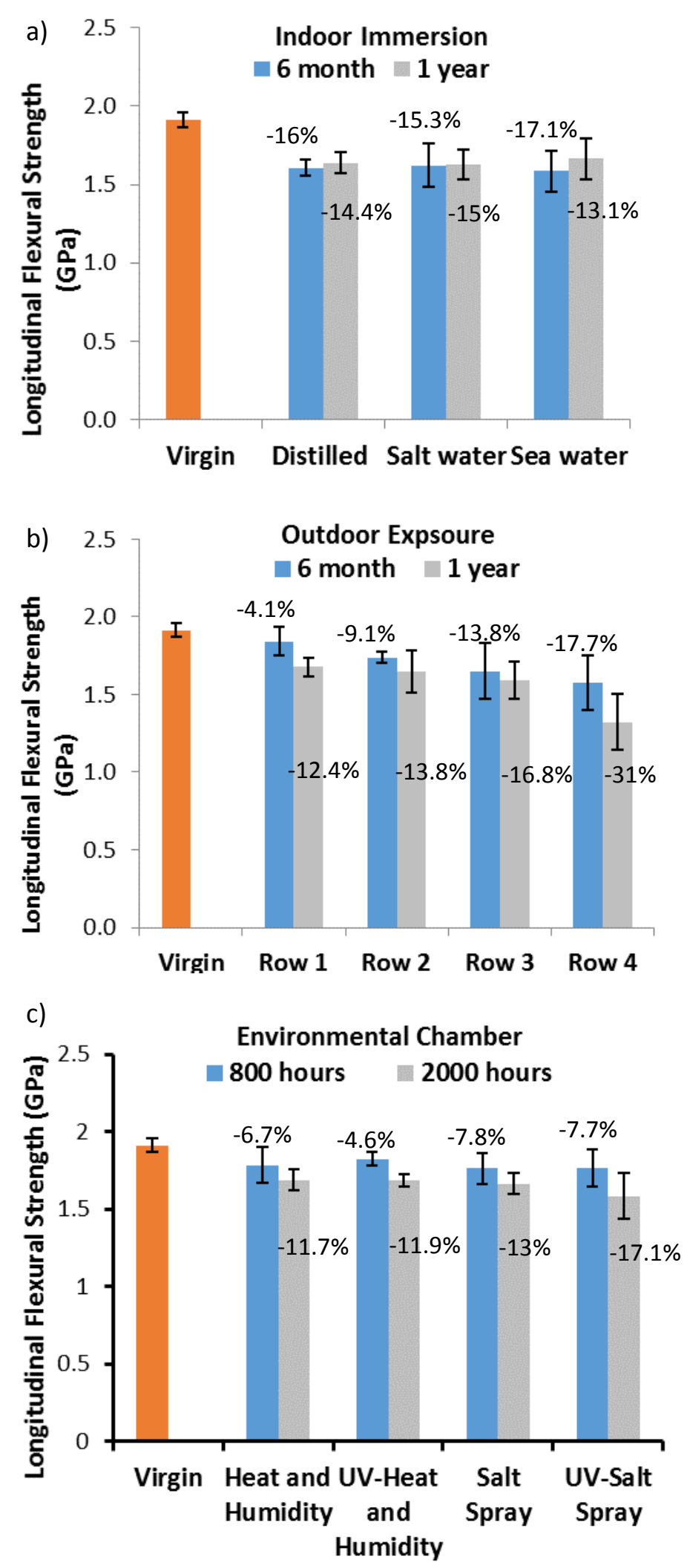

Figure 3: Longitudinal flexural strength of CFVE composites exposed to (a) Indoor immersion, (b) Outdoor environmental exposure, and (c) Conditioning in environmental chambers 


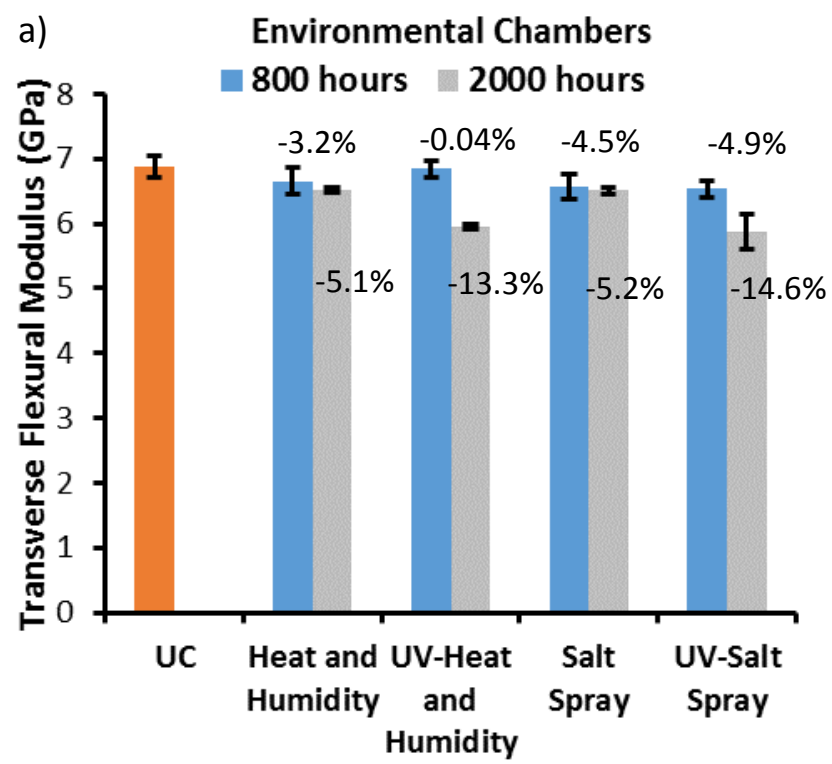

b)

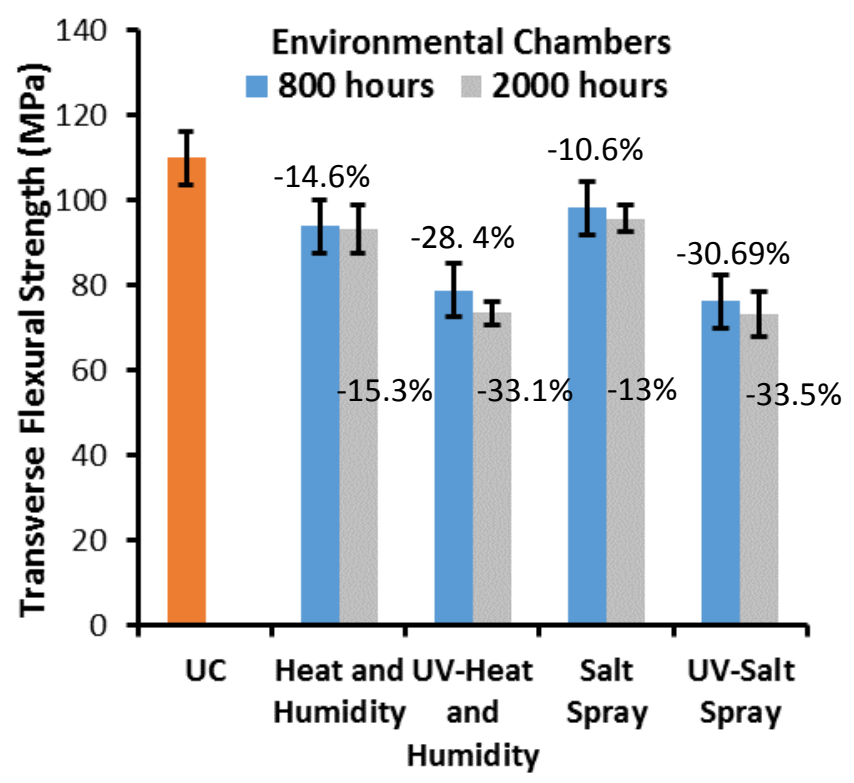

Figure 4: Transverse flexural properties of CFVE composites conditioned in Environmental chambers (a) Flexural modulus, (b) Flexural strength. (UC $=$ Unconditioned specimens) 


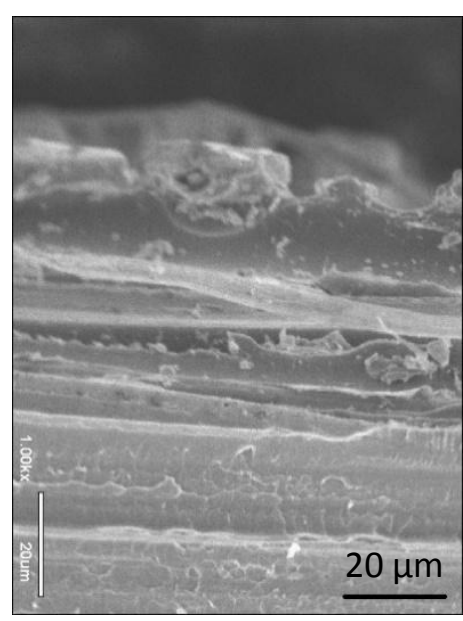

(a)

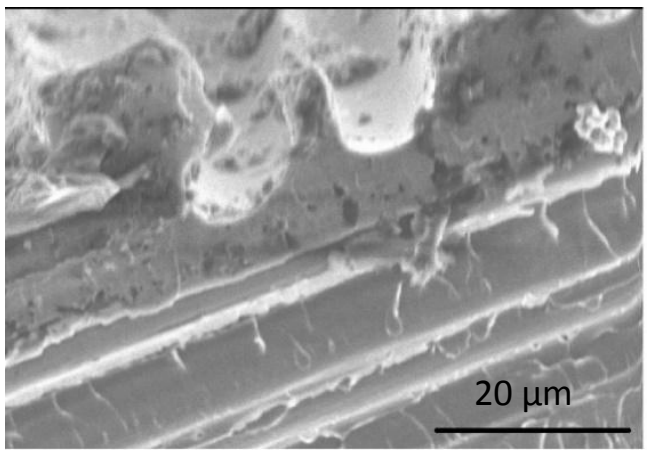

(c)

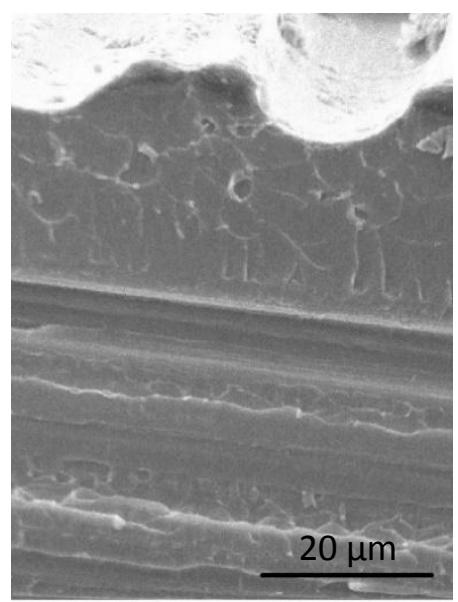

(b)

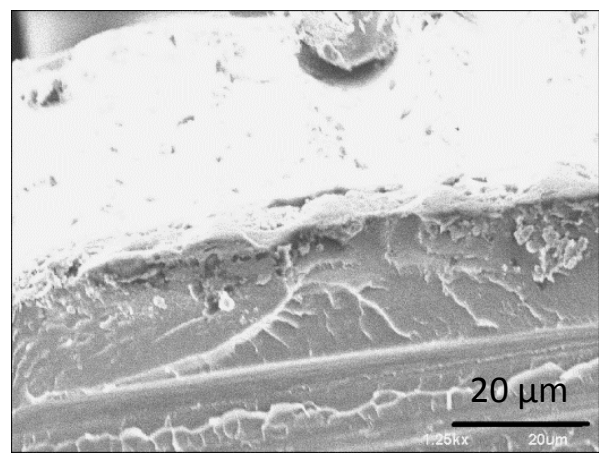

(d)

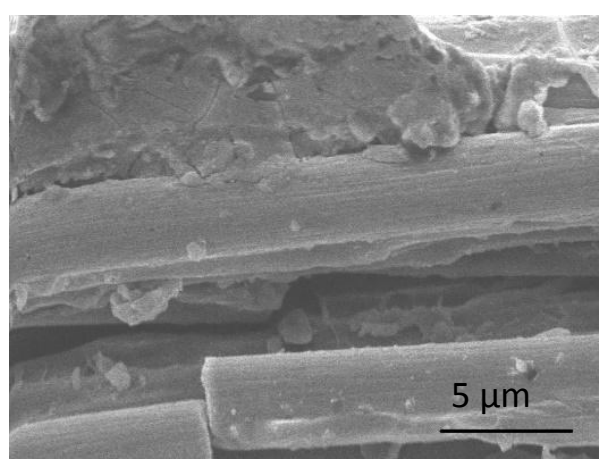

(e)

Figure 5: Fracture surface of $\left[90^{\circ}\right]$ specimens after 2000 hours exposure to (a) no conditioning (b) salt spray (c) heat and humidity (d) combined UV, heat and humidity (e) combined UV, heat and humidity (magnified) 